\title{
La mimesis : aspects ludiques et poétiques
}

\section{Michael Groneberg}

\section{(2) OpenEdition}

\section{Journals}

Édition électronique

URL : http://journals.openedition.org/edl/1092

DOI : 10.4000/edl.1092

ISSN : 2296-5084

\section{Éditeur}

Université de Lausanne

\section{Édition imprimée}

Date de publication : 15 mars 2018

Pagination : 145-168

ISBN : 978-2-940331-67-3

ISSN : 0014-2026

\section{Référence électronique}

Michael Groneberg, «La mimesis : aspects ludiques et poétiques », Études de lettres [En ligne], 1 | 2018, mis en ligne le 15 mars 2020, consulté le 11 décembre 2020. URL : http://journals.openedition.org/ edl/1092 ; DOI : https://doi.org/10.4000/edl.1092 


\section{LA MIMESIS : ASPECTS LUDIQUES ET POÉTIQUES}

Sous la forme d'un commentaire de quelques propos centraux de Denis Guénoun sur le théâtre, cette contribution discute les possibles acceptions du terme "mimesis", comprise entre autres comme jeu et comme expression chez Platon et Aristote. Car c'est bien dans le jeu mimétique et non dans une activité de reproduction ou de copie que réside la valeur cognitive et poétique du drame. Dans le même mouvement, la discussion de ces termes mettra en lumière une certaine conception post-aristotélicienne du texte théâtral et du jeu scénique, conception qui leur accorde une place centrale dans notre vie commune.

Dans Le théâtre, est-il nécessaire? paru en 1997, Denis Guénoun distingue trois univers différents dans l'histoire du théâtre occidental et y identifie ce que j'appellerai des "régimes" théoriques. Ces trois univers se trouvent, respectivement, dans l'Athènes classique $\left(\mathrm{V}^{\mathrm{e}}\right.$ au IV $\mathrm{V}^{\mathrm{e}}$ siècle av. notre ère), dans le classicisme français (XVII ${ }^{\mathrm{e}}$ et XVIII ${ }^{\mathrm{e}}$ siècle) et dans le théâtre moderne du $\mathrm{XX}^{\mathrm{e}}$ siècle. Les régimes qu'il propose de distinguer sont, dans cet ordre, le mimétique, le représentatif et celui du jeu. Ils sont décrits successivement dans des chapitres non-titrés: le premier (p.17-42) se base sur la Poétique d'Aristote et le deuxième (p. 43-81) sur des auteurs du classicisme français (de d'Aubignac à Diderot, en passant par Sainte-Albine et Riccoboni). Celui qui nous expose, in fine, le régime contemporain du jeu est le chapitre V (p. 143 sq. $)^{1}$.

I. Il est précédé par deux chapitres; le premier (ch. III) traite de la scène psychanalytique chez Freud (p. 83-95) et Stanislavski (p. 96-101) et le deuxième (ch. IV) montre comment le cinéma réalise d'anciens rêves du théâtre, le contraignant à identifier et à focaliser sur ce qui lui est propre (p. 103-141). 
Guénoun nous propose une sorte de Dreischritt dialectique ${ }^{2}$ dont le troisième pas est modifié par l'arrivée du cinéma. Il nous mène de l'indifférenciation entre représentant et représenté, entre comédien et personnage, à une distinction claire et nette des deux pour aboutir enfin à un dépassement qui rend la différence inessentielle: le représenté n'est plus nécessaire au spectacle. Or, la séparation du représenté et du représentant, du signifié et du signifiant, typique de l'époque classique, ne serait pas encore en fonction dans le théâtre antique: "la mimèsis aristotélicienne est relativement indifférente à l'opposition entre la figure et son référent, voire qu'elle est construite, précisément, sur la position de cette indifférence» (p. 21) et ne serait plus pertinente à notre époque où l'on s'intéresse désormais au jeu en lui-même, sans qu'il ne renvoie à une action ou à un personnage au-delà ou derrière lui : "sur scène, désormais, ne reste que le jeu [...] sa nécessité intrinsèque ne se déduit plus du besoin de donner vie à des personnages» (p. 144 sq.). L'« exigence n'est pas représentative, mais présentative plutôt» (p. 147). Le théâtre actuel ne serait plus à comprendre comme représentation dans le sens où le jeu représenterait une action pré-existante, ni l'acteur un personnage pré-existant. Parmi les pistes que Guénoun esquisse, pour décrire positivement ce jeu et sa justesse, figure éminemment la poésie: «La vérité du texte théâtral est désormais, intempestivement, poétique» (p. 148).

Ces quelques mots doivent suffire à reproduire ici la position énoncée par Guénoun il y a vingt ans. Le commentaire suivant rappellera quelques éléments éclairants de son analyse, en amenant pourtant un questionnement de ses conclusions générales par l'argument que le texte théâtral aussi bien que le jeu ont toujours été poétiques dans un sens qui est aussi porté par le terme "mimesis». Pour ce faire, je propose une analyse des termes utilisés par Platon et Aristote pour signifier le jeu, dont celui de «mimesis» qui joue la figure centrale.

2. On utilisera ce terme original qui est difficilement traduisible - on a proposé «triade dialectique» - propre à la philosophie dialectique. Littéralement «trois pas», il signifie l'avancement en trois étapes (comme thèse, antithèse, synthèse ou indifférence, différence, réconciliation). 


\section{Platon: la République}

Dans la critique de l'art en général et de la mimesis en particulier énoncée dans la République de Platon, nous trouvons trois arguments contre la poésie. Le premier, dans les livres II à III, présente la construction, «en pensée» (logôi, Rép. II, 369a7), d'une cité dans laquelle il s'agira d'identifier la justice et l'injustice. Après l'introduction de la classe des gardiens, il s'avère nécessaire de discuter leur éducation, non seulement pour former de bons guerriers pleins de ferveur, mais également pour éviter tout abus de pouvoir desdits gardiens. L'éducation commençant par les histoires que les mères et nourrices racontent aux enfants, il faut, dit Socrate, surveiller leur contenu et les poètes qui les créent. Après avoir énuméré toutes sortes de contenus qui seraient à supprimer dans les contes des poètes, Socrate pose enfin la question de la présentation du contenu admissible:

[Socrate] Tout ce que disent les conteurs de fables et les poètes [hupo muthologôn è poiètôn legetai], n'est-il pas le récit d'événements passés, présents ou futurs [diègesis ousa tunchanei è gegonotôn è ontôn è mellontôn] ? - [Adimante] Comment, répondit-il, serait-ce autre chose? - Eh bien! n'emploient-ils pas pour cela soit le récit simple [haplèi diègesèi], soit la mimèsis de ce qui arrive [dia mimèseôs gignomenèi], soit l'un et l'autre à la fois? $(392 \mathrm{~d} 2-5)^{3}$

Ces trois formes diégétiques sont explicitées plus loin. Il s'agit, dans l'ordre, de la poésie dithyrambique qui est un récit simple, de la tragédie et la comédie composées seulement de paroles sans récit simple et de l'épopée qui mélange récit et mimesis (l'exemple utilisé étant l'Iliade). La mimesis y est définie comme présentation d'un discours "comme si le poète était un autre" (hôs tis allos ôn, 393c1), par exemple quand Homère, après avoir commencé l'Iliade avec un récit simple, nous raconte, "comme s'il était devenu Chrysès", comment ce dernier vient demander la libération de sa fille.

Constatons que l'approche de Platon est narratologique: il traite le poème, le drame et l'épopée comme des narrations (diègeseis) à l'intérieur

3. Trad. R. Baccou (p. 144), légèrement modifiée. Voir aussi l'opposition entre mimesis et récit simple (haplè diègesis) en 393d6. Socrate y donne un exemple de récit simple qui n'est autre qu'un rapport descriptif de ce qui s'est passé devant Troie. 
desquelles il introduit une distinction fondamentale entre narration simple et mimesis. Cette dernière peut, à cet endroit, être traduite aussi bien par "jeu mimétique» que par «imitation" ou "reproduction». Notons cependant que le terme dénote tout d'abord un certain genre de texte. Ce n'est que plus loin dans la République qu'il désigne aussi le jeu imitatif des gardiens, et plus tard encore, dans le livre X, qu'il sera question d'une mimesis dans un sens plus large, couvrant aussi la mimesis des peintres.

De quelles paroles et actions est-il question dans le passage cité? L'interlocuteur, Adimante, ne rend-il pas le jeu dialogique trop facile pour Socrate? Qui pourrait soutenir que les poètes racontent toujours ce qui s'est vraiment passé? Qui n’aurait pas envie de dire: "Mais non, Socrate, je suppose bien que les faiseurs de fables racontent souvent ce qui ne s'est jamais passé et n’arrivera jamais »? A l'époque de Platon, le poète est déjà libéré du défi de raconter des faits, tâche qui incombe désormais aux historiographes qui cherchent à écrire le passé et aux philosophes qui se préoccupent des généralités du savoir sur le monde 4 . Platon le sait bien: il invente lui-même des récits, qu'il s'agisse de la mort de Socrate, du banquet dans la maison d'Agathon ou des mythes de toutes sortes qui foisonnent dans ses dialogues. Aristote le constatera:

[...] la poésie est plus philosophique et plus noble ${ }^{5}$ que l'Histoire [philosophôteron kai spoudaioteron poièsis historias estin] : la poésie dit

4. Cf. M. Puelma, Der Dichter und die Wahrheit in der griechischen Poetik von Homer bis Aristoteles. Dans la mesure où le passé commun n'est pas écrit mais chanté, ce ne sont pas non plus les tragédiens qui accomplissent cette tâche, mais les aèdes et rhapsodes qui chantent les épopées, comme D. Bouvier l'explique dans sa contribution.

5. Ce terme spoudaios qui signifie initialement "s'appliquer avec sérieux", "être de bonne qualité", mérite notre attention. La spoudè exprime hâte, effort ou zèle et s'oppose comme l'effort au loisir (scholè) et comme le zèle au jeu (paidia). Il s'agit, comme l'a mis en évidence A. Schniewind (L'éthique du sage, p. 30-35), d'un terme éthique qui caractérise l'homme de bien qui possède la vertu. La spoudè s'applique d'abord au héros grec qui agit avec prudence. Elle caractérise chez Platon le philosophe qui s'implique dans le monde et chez Aristote, l'homme bon ancré dans le monde indépendamment de son statut social. Chez Platon, le sérieux et le noble de la spoudè s'opposent au ludique (paidia) - en particulier aux jeux d'enfants qui sont pourtant à prendre très au sérieux, car ils préparent au sérieux de l'âge adulte; moins paradoxalement, le spoudaios s'oppose aussi au bas, vil ou vulgaire (phaulos). Chez Aristote, la tragédie est définie comme mimèsis praxeôs spoudaias kai teleias (Poétique 6, 1449a24), donc comme «jeu mimétique d'une action sérieuse et achevée»; le contraire en est le phaulon, le bas ou 
[legei] plutôt l'universel [ta katholou], l'histoire le singulier [ta kath'hekaston] (Aristote, Poétique 9, 1451b6-7, trad. MG).

Platon met en scène un Adimante plus naïf que lui-même. S’il est vrai de dire que le personnage de Socrate dans ses dialogues mime le Socrate historique, il n'est pas nécessaire de supposer qu'il l'imite ou qu'il le reproduit ou le représente. Dans le passage cité d'Aristote, la relation entre la poésie et l'universel est de l'ordre du legein, du dire ou de l'expression, selon la traduction de Barbara Gernez. La mimesis propre à la poésie dit ou exprime donc des généralités - et l'on peut supposer que Platon le sait, contrairement aux personnages qu'il met en scène. Dans l'acception qui apparente la mimesis à la parole exprimant une généralité (et qui ne copie pas une autre parole individuelle), celle-ci acquiert un statut plus noble qu'une mimesis qui ne serait que la simple reproduction sous le même mode (des paroles parlées qui copient des paroles entendues, des images peintes qui reproduisent des images vues etc.).

Platon s'efforce de définir la mimesis dans les lignes que nous avons citées comme tentative de correspondre à celui qu'on prétend être ${ }^{6}$, en utilisant ici un terme, homoioun (393c), qui servira à Aristote pour exprimer ce que l'on connait aujourd'hui en sémantique sous le nom de "principe de correspondance». Ce terme renvoie à la relation particulière entre un événement et une phrase qui fait que la phrase parlant de l'événement est vraie. Pour qu'une phrase soit vraie, il faut qu'elle soit homoioun avec le fait dont elle parle, qu'elle lui corresponde ${ }^{7}$. Cette correspondance n'est pas une similarité ou une ressemblance, car phrase et événement ne sont pas semblables ou similaires (ils n'existent pas sur le même mode), comme les traductions du grec peuvent parfois nous le faire croire.

le vulgaire de la comédie (cf. Poétique 2, 1448a1-2; 5, 1448b33). Il n'est pas seulement question de la mimesis d'actions nobles, mais aussi de celle de personnes nobles ou "plus nobles (ou meilleures) que nous" (mimèsis estin hè tragoidia beltionôn è hèmeis, Poétique 15, 1454b8), ce qui caractérise la tragédie mais aussi les épopées d'Homère (Poétique 3, 1448a28); la comédie par contre est mimèsis phauloterôn (Poétique 5, 1448b32), mimétique donc de ceux qui sont plus vils.

6. R. Baccou traduit: «se rendre semblable à un autre sous le rapport de la voix et de l'aspect, c'est imiter celui auquel on se rend semblable» (393c5).

7. Aristote, De l'interprétation, ch. 9, 18b7-8, 19a32. 
La mimesis ne signifie donc pas forcément une relation d'empreinte, de modèle et copie, de simulation ou de reproduction, mais peut dénoter la transposition dans un autre mode. Il s'agit bien d'une relation de référence (du point de vue du produit) ou d'expression (du point de vue de l'artiste) ${ }^{8}$. Cette «relation d'expression" n'implique pas de similarité formelle (de même Gestalt), au contraire de la relation de simulation, de copie ou de reproduction en laquelle on peut rêver une imitation parfaite qui rendrait produit et original indiscernables. La compréhension habituelle de la mimesis comme imitation qui s'apparente à la simulation est une bonne raison pour chercher une autre traduction du terme chez Platon et Aristote. Nous devons tenir compte du fait qu'il peut s'agir d'une relation comparable à la correspondance d'une phrase au fait dont elle parle. La description d'une action dans un texte peut correspondre plus ou moins à l'action décrite (si elle a existé), mais elle lui ressemble aussi peu qu'un énoncé à un événement ${ }^{9}$.

Dans le livre III de la République, Socrate et son interlocuteur Adimante sont d'accord pour dire que la mimesis - dans son sens étroit - est à comprendre comme «faire comme si on était un autre». On pourrait croire qu'il est ici question du jeu du comédien qui simule, avec son action, celle d'un autre. Mais la référence est tout d'abord textuelle: Platon évoque le supposé aède Homère qui dans l'écriture passe du récit au jeu de paroles. La mimesis, même dans son sens étroit, a donc une double signification: celle du jeu dans le texte poétique - où l'auteur parle comme s'il était un autre -, et celle du comédien sur scène, ou de l'enfant dans la cour qui joue un rôle. Pour distinguer cette sorte de jeu des jeux non-mimétiques de société, de sport, d'enfants et autres, il parait judicieux de traduire la mimesis au sens étroit par "jeu mimétique», signifiant aussi bien l'interaction décrite par un auteur que celle réalisée par les acteurs. Le terme "mimétique» dans son sens large, par contre, englobe encore, chez Platon et Aristote, la mimesis de la peinture,

8. Nous disons aussi, à juste titre, que les gênes s'expriment dans un environnement donné. C'est dans ce sens que la volonté se trouve exprimée, selon Schopenhauer, directement par la musique, sans avoir besoin de représentations (Le monde comme volonté et comme représentation, \$52).

9. L'analyse de J.-M. Schaeffer des types de mimétismes néglige ce type d'expression (cf. Pourquoi la fiction?, p. 64 ss., en particulier les trois types décrits aux p. 80 sq.). C'est pourquoi elle ne saisit pas complètement le concept antique de mimesis. 
de la sculpture et de la danse, et celle de tout art poétique, y compris de la narration pure.

Pour revenir à la République, nous savons que la mimesis y est présentée comme dangereuse pour les jeunes gardiens de la cité - et il s'agit d'abord de jeux mimétiques:

[Socrate:] Ou bien n'as-tu pas remarqué que les jeux mimétiques [hai mimèseis, pluriel], si depuis l'enfance [ek neôn] on persévère à les cultiver, se fixent dans les habitudes et dans la nature du corps, de la voix et de l'esprit? (395d1-2; trad. MG)

Si donc ces enfants jouent, ils ne devraient mimer que la vertu. On interdira aux futurs gardiens, s'ils sont des hommes, de jouer une femme ou un homme méchant ou faible, et ainsi de suite. Ici, la mimesis est à comprendre comme une reproduction par simulation, semblable à la copie, et ayant un aspect contagieux, transmettant et fixant ce qui est simulé dans le simulateur.

Cet argument pédagogique du livre III sera repris plus loin, au début du livre X, et renforcé par deux arguments supplémentaires. Le premier est psychologique et traite du rapport de force entre la raison et les passions dans l'âme du spectateur au théâtre. Le second est métaphysique et concerne la distance de la vérité. Le personnage Sôkratès y renforce la critique des poètes en transformant l'argument de la censure en une justification du bannissement des poètes. C'est en particulier l'art mimétique, au sens étroit, qui est la cible de ces critiques, mais Socrate commence à nouveau par une définition de la mimesis, cette fois en utilisant la métaphore de l'artisan qui produit un lit, pour distinguer ensuite trois sortes de création (poièsis au sens large; 595c7) : celle du dieu qui a créé l'idée ou la forme du lit; celle de l'artisan qui produit les lits suivant cette forme; et enfin celle du peintre qui peint des images de lit en s'inspirant - de quoi? Arrive, à cet endroit précis, la Gretchenfrage ${ }^{10}$ à l'origine de toute théorie de l'art:

[Socrate:] Lequel des deux buts poursuit la peinture par rapport à chaque chose: d'exprimer [mimèsasthai] ce qui est tel qu'il est, ou ce

Io. La question la plus décisive ou la plus cruciale; la question clé. Gretchen demande à Faust dans le drame éponyme de Goethe: Nun sag, wie hast du's mit der Religion? 
qui paraît tel qu'il paraît? Est-elle la mimèsis de l'apparence ou de la vérité [phantasmatos è alètheias ousa mimèsis]?

[Glaucon:] De l'apparence (Platon, Rép. X, 598b1-4; trad. MG).

Voilà deux relations bien distinctes qui sont signifiées par la mimesis: la reproduction de l'image d'un lit, comme il apparaît dans notre perception, par une image créée par le peintre, et la réalisation d'un concept, l'expression - dans la matière perceptible - d'une entité abstraite et nonperceptible.

Notons qu'à ce carrefour du dialogue, Socrate formule clairement les deux options et lance ainsi le défi d'une réponse inverse. La bifurcation est fondamentale. Car peu importe la réponse: accepter de répondre à cette question implique accepter la présupposition selon laquelle l'art représenté ici par la peinture - est à comprendre par sa proximité, ou sa distance, à la vérité: la question lie l'art conceptuellement à la vérité. La ligne de démarcation débattue est plus décisive que le côté duquel on est situé. Le manœuvre a bien réussi à enfermer la conception de l'art dans un discours de vérité. En fin de compte, la question posée ici par Socrate a trouvé, dans l'histoire de la théorisation de l'art, la réponse alternative qui n'était pas choisie par Glaucon. S'il est juste de dire qu'avec sa République, Platon a mis en scène l'accusation de la poésie, il est faux de dire qu'il aurait banni le théâtre de la cité. Socrate présente trois arguments contre elle dont il souhaite explicitement qu'ils soient réfutés:

[Socrate] Déclarons néanmoins que si la poésie peut nous prouver par de bonnes raisons qu'elle a sa place dans une cité bien policée, nous l'y recevrons avec joie, car nous avons conscience du charme qu'elle exerce sur nous - mais il serait impie de trahir ce qu'on regarde comme la vérité. Autrement, mon ami, ne te charme-t-elle pas toi aussi, surtout quand tu la vois à travers Homère?

[Glaucon:] Beaucoup.

[Socrate] Il est donc juste qu'elle puisse rentrer à cette condition: après qu'elle se sera justifiée, soit dans une ode, soit en des vers de tout autre mètre.

[Glaucon] Sans doute.

[Socrate] Nous permettrons même à ses défenseurs qui ne sont point poètes, mais qui aiment la poésie, de parler pour elle en prose, et de nous montrer qu'elle n'est pas seulement agréable, mais encore utile au gouvernement des États et à la vie humaine; et nous les écouterons 
avec bienveillance, car ce sera profit pour nous si elle se révèle aussi utile qu'agréable.

[Glaucon:] Certainement nous y gagnerons (Platon, Rép. X, 607c-d).

Aristote relève ce défi et y répond, dans sa Poétique, en légitimant le drame ${ }^{11}$. Un demi-millénaire plus tard, Plotin formule la défense générale qui restera la base de la conception de l'art jusqu'aux temps modernes:

Et si quelqu'un méprise les arts sous prétexte que c'est en imitant la nature qu'ils produisent [hoti mimoumenai tèn phusin poiousi], il faut d'abord lui dire que les réalités naturelles sont elles aussi des imitations [tas phuseis mimeisthai alla]; ensuite il faut qu'il sache que les arts ne se bornent pas à imiter ce qu'on voit, mais qu'ils sont à la poursuite des raisons [epi tous $\operatorname{logous}^{12}$ ] dont est faite la nature ${ }^{13}$.

Le père du néo-platonisme emprunte l'autre voie qu'ouvrait Platon dans la République. Qu'il s'agisse d'une provocation ou d'un défi lancé, Platon l'a bien réussi. Il a, en tout cas, eu pour effet d'enfermer toute conceptualisation de l'art dans un discours de proximité à la vérité conçue comme idée éternelle. Mais c'est justement ici qu'Aristote offre une ouverture, comme nous le verrons plus loin.

Remarquons d'abord deux autres termes figurant dans le champ lexical de la mimesis chez Platon:

II. Le mot drama est attesté chez Aristote qui explique que tragédie et comédie ont en commun de mimer des agissants (Gernez traduit: "elles imitent des exécutants", p. 11 et n. 10), et c'est pourquoi elles sont appelées dramata (Poétique 3, 1448a28; le mot est dérivé du verbe dorien dran pour agir). Dans les passages sur la tragédie, la défense contre l'attaque psychologique par le biais de la catharsis est explicite, mais il est aussi légitime de parler, par extension, de la défense du drame. Puisque le traitement de la comédie est perdu, nous ne savons pas si Aristote a construit un autre type de catharsis opérant par d'autres émotions, pour défendre la comédie et son effet, le rire. Par contre, ce n'est pas seulement la tragédie, mais la poésie tout entière, la comédie comprise, qui est défendue comme "disant l'universel», comme étant "plus noble et plus philosophique» (cf. le passage cité ci-dessus).

I2. Chez Plotin, ces logoi ne sont pas les idées en elles-mêmes, mais les idées comme elles se présentent à l'homme, leurs rejetons dans l'âme humaine (cf. L. Brisson, "Logos et logoi chez Plotin»).

I3. Plotin, Traité 31 (V, 8), 1, 33-35 (trad. L. Brisson). Bréhier traduit mimoumenai $[. .$.$] poiousi par "créent des images", comprenant ainsi la mimèsis comme étant de$ l'ordre de l'imagerie ou de l'empreinte (Plotin, Ennéades, V 8 [31], 1, 33-35). 
[Socrate] Voilà donc, ce semble, deux points sur lesquels nous sommes bien d'accord: tout d'abord l'imitateur [mimètikos] n'a aucune connaissance valable de ce qu'il imite, et l'imitation n'est qu'une espèce de jeu d'enfant, dénué de sérieux [all'einai paidian tina kai ou spoudèn tèn mimèsin] (Platon, Rép. 602b; trad. Baccou).

Ce qui paraît ${ }^{14}$ clairement dépréciatif à cet endroit - la République poursuit une logique étatique visant le bien communautaire -, l'est moins ailleurs. Dans le Banquet - porté au contraire par une logique de l'individu -, Platon met dans la bouche d'Agathon (qui vient de gagner un concours de tragédie "devant 30'000 spectateurs»; Banquet 175e) un éloge de l'amour (éros) qui se termine par une auto-caractérisation comme "discours qui, autant que faire se peut, participe de façon mesurée aussi bien au jeu qu'au sérieux" (ta men paidias, ta de spoudès metrias) ${ }^{15}$. Voici mis en opposition le ludique et le sérieux. Les deux semblent pourtant réconciliables dans le discours d'un écrivain de tragédies...

Retenons que Platon utilise le terme «mimesis» d'abord (au sens étroit) pour désigner le mode textuel "dramatique», puis le jeu mimétique des enfants et des adultes dans l'intention de reproduire les histoires héroïques mais qu'ensuite, il l'utilise aussi (au sens large) pour parler de la production du peintre et du sculpteur. Ajoutons que, telle qu'il la définit dans au moins un de ses "dialogues socratiques" exotériques (destinés à être lu par un public large à l'extérieur de son école), cette mimesis (au sens large) est également dénoncée comme manquant systématiquement de sérieux, ludique comme un jeu enfantin.

\section{Aristote: la Poétique}

La Poétique peut être conçue comme étant, en premier lieu, une réponse d'Aristote au défi lancé par son maître qui invitait à trouver une légitimation à la poésie dramatique. Le disciple le fait en accordant une légitimité anthropologique et épistémologique à la mimesis, en réduisant l'importance du spectacle et en accordant la première place

I4. Il pourrait s'agir d'une autre provocation.

15. Platon, Banquet, 197e, trad. de L. Brisson modifiée. 
à l'intrigue qui contient, selon lui, l'essentiel du drame - celle-ci est sa «fin" (telos, 1450a23) ou "comme son âme" (hoion psuchè, 1450a38). De plus, il justifie les effets du drame sur le lecteur ou le spectateur comme cathartiques, purifiants, rétablissant le gouvernement de l'intellect sur les passions.

Il est pourtant erroné de croire que la katharsis serait le noyau théorique et l'invention originelle d'Aristote, ce que les discussions depuis la Renaissance tendent à nous faire croire. D'une part, l'analyse des effets émotionnels chez les spectateurs ne diffère guère de celle de Gorgias, sinon en ce que le regret y est supprimé, ou plutôt absorbé par la pitié:

Par elle [la poésie], les auditeurs sont envahis du frisson de la crainte, ou pénétrés de cette pitié qui arrache les larmes ou de ce regret qui éveille la douleur, lorsque sont évoqués les heurts et les malheurs que connaissent les autres dans leurs entreprises; le discours provoque en l'âme une affection qui lui est propre ${ }^{16}$.

D'autre part, émerge ici une question toute légitime: si utile que l'argument du nettoyage des passions puisse être pour justifier le théâtre, est-ce vraiment une description juste de ce qui arrive au spectateur qui assiste à une tragédie de Sophocle dans l'Athènes au Ve siècle av. notre ère, ou aux âmes des lecteurs de drames un siècle plus tard ${ }^{17}$, voire à la spectatrice au théâtre moderne?

Catharsis ou purgation [...] Nous ne sommes pas au cœur du sujet. Quand le rideau se lève au théâtre, personne, jamais, n’a songé à la catharsis. Avec sa purgation, Aristote n'est que le pharmacien du théâtre. C'est un poète lorsqu'il découvre que le texte a une âme. Il y a plus de profondeur dans cette constatation que dans le système de la catharsis ${ }^{18}$.

Aristote pharmacien du théâtre? Disons plutôt: Aristote bon avocat de sa défense. Or, pourquoi veut-il défendre le drame, lui qui accorde tout de même sa préférence à la sagesse théorique, la sophia, qu'il juge

I6. Gorgias, DK 82 B11, 9; trad. Dumont p. 712 (LM 32 Gorgias D24).

17. Eschyle, Sophocle, Euripide et Agathon ont tous écrit au Ve siècle - la Poétique est écrite dans la deuxième moitié du IV ${ }^{\mathrm{e}}$, avant la mort d'Aristote en 322.

I8. L. Jouvet. Témoignages sur le théâtre, p. 248 sq. 
supérieure à la sagesse pratique, la phronèsis ${ }^{19}$ ? Si la Poétique est plus une défense contre l'accusation platonicienne qu'une description basée sur l'observation, quels éléments ont bien pu amener Aristote à monter un tel plaidoyer?

A mon sens, les drames formulent pour Aristote le savoir dans le royaume de la contingence, cette sagesse pratique ayant la particularité de n'être jamais parfaite ${ }^{20}$. Sans pouvoir expliquer ce point en détail ici, il suffit de voir que, dans la perspective aristotélicienne, la science et le savoir ont pour objet le nécessaire: ce qui ne peut être changé, c'està-dire le passé (domaine de l'historiographie) et ce qui est de manière atemporelle, comme les lois mathématiques et logiques ou ce qui arrive nécessairement (domaine des sciences, comme de la physique $;^{21}$ on dirait aujourd'hui: le domaine des lois de la nature). Le terme "contingent", au contraire, désignera ce qui peut arriver et peut également ne pas arriver $;^{22}$ en d'autres mots: ce qui n'est ni nécessaire ni impossible. Ce concept de la double potentialité d'une chose joue un rôle central dans l'ontologie aristotélicienne de l'action humaine. La contingence est, pour Aristote, une condition nécessaire de toute action. Pour qu'un homme soit responsable de son acte, il faut qu'il ait pu s'en abstenir ${ }^{23}$. Si tout était nécessaire, il n'y aurait pas d'actions.

Or, si ce que nous ferons demain peut aussi ne pas être fait, il n'y a pas (encore) d'énoncé vrai ou faux qui puisse porter sur cette action. Il n'y a donc pas de savoir possible par rapport à une action future. Bien sûr, il existe un savoir éthique qui nous dit comment nous devrions agir pour devenir heureux; un savoir qui devrait, selon nos auteurs et leurs successeurs, imposer ses commandements par le biais des lois et de l'éducation à ceux qui n'ont pas le loisir ou les compétences d'y réfléchir ${ }^{24}$. Il $\mathrm{y}$ a pourtant aussi un savoir descriptif des actions libres des hommes, et

19. Aristote, Éthique à Nicomaque, X.7, 1177a11 sq.

2o. Ibid., VI.5, 1140b22.

2I. Ibid., VI.2-6, 1139a1-1141a9.

22. Ibid., VI.4, 1139b36-1140a1; De l'interprétation, ch. 9, p. ex. 19a3; Métaphysique, Theta 4. Le terme est plus tardif, Aristote utilise le concept sans le nommer.

23. Aristote, Éthique à Nicomaque, III, 1113 b7-8. Voir aussi De l'interprétation, ch. 9 .

24. Cf. Éthique à Nicomaque, 10, $1179 \mathrm{~b} 4$ sq. 
c'est ce savoir qui est le domaine des poètes. ${ }^{25}$ Ici se trouve sans doute la raison de la défense aristotélicienne du drame: il formule un savoir sur nos actions libres - ce qui peut, à première vue, paraître paradoxal. Cela l'est moins si nous considérons que même des actions individuellement libres peuvent surgir les structures de nos interactions.

Si l'on suppose que l'objectif défensif prime chez Aristote sur celui de l'adéquation de sa description avec les drames auxquels il se réfère, il est d'autant plus frappant de voir que son plaidoyer ne prend pas appui sur une base narratologique, comme chez Platon, mais sur une base mimétologique ${ }^{26}$ :

L'épopée donc et la poésie tragique, ainsi que la comédie, la poésie dithyrambique et, pour une bonne partie, l'art de la flute et celui de la cithare, ont tous ceci de commun qu'ils sont, de manière générale, des imitations [mimèseis ${ }^{27}$.

On connait bien le reproche fait au "vampire du théâtre occidental» selon lequel il aurait fait dominer le texte sur le spectacle ${ }^{28}$. Cependant, n'est-ce pas surprenant qu'il prenne, contrairement à son maître, appui sur un assemblage de formes d'art vivant, et non sur le texte?

Toutefois, loin de signifier seulement le jeu du comédien, chez Aristote aussi, la mimesis couvre également les descriptions.

[...] on peut imiter [mimeisthai] les mêmes affaires en les rapportant [apangellonta], [a] soit en devenant un autre, comme Homère le fait, [b] soit en restant le même et sans transformation [mê metaballonta], [c] soit tout en agissant et en actualisant ce qui est à imiter [ê pantas hôs prattontas kai energountas tous mimoumenous] (Poétique 3, 1448a21-24, trad. MG).

25. Guénoun, dans la partie de son texte qui porte sur la Poétique, analyse aussi la valeur cognitive du théâtre en se focalisant sur quelques lignes de ce texte traitant la (re)connaissance, comportant le "celui-là, c'est lui!» (D. Guénoun, p. 28; Aristote, Poétique, 1448b15-17).

26. On devrait, à mon avis, tester l'hypothèse que ce soit justement cela qui permette de trouver une issue aux trois problèmes (pédagogique, psychologique et métaphysique) formulés par Platon.

27. Aristote, Poétique 1, 1447a13-15.

28. Cf. F. Dupont, Aristote ou le vampire du théâtre occidental. 
Aristote en arrive alors à une distinction en trois genres identique à celle que Platon avait proposée:

a. le mode mixte (récit et jeu) : récit et jeu mimétique dans les épopées, comme l'Iliade,

b. le récit sans jeu (dithyrambes),

c. le jeu mimétique complet (tragédie, comédie).

Les mots (écrits ou parlés) font partie de l'un des trois moyens de la mimétique (ch.1, 1447a22: «rythme, langage et mélodie», b25: "rythme, chant et mètre»). Les objets de la mimétique sont des actions, des caractères et des pensées, divisés en deux catégories: nobles (dans la tragédie) ou bas (dans la comédie) ${ }^{29}$. Finalement, Aristote distingue les deux manières d'imiter, à savoir en racontant (par la diègèsis) et en montrant (par la mimesis), reproduisant ainsi l'acception double du terme mimesis qui, au sens étroit, désigne le jeu mimétique - qu'il soit écrit ou mis en acte - et, au sens large, l'expression d'actions par leur narration, leur reproduction dans la danse ou par l'intermédiaire d'une mélodie jouée à la flûte.

Aristote déplace ainsi la base d'analyse de la narration d'événements à la mimesis d'actions. Nous assistons ainsi à un déplacement de la diégèsis à la mimesis comme activité fondamentale, mais aussi des événements aux actions, ce qui est crucial. Même si l'imitation peut avoir un objet autre que les actions: «les danseurs imitent [...] les caractères, les émotions et les actions» (Poétique 1, 1447a27-28), et même si la tragédie est "mimèsis non pas d'hommes, mais d'action et de vie et de bonheur» (6, 1450a17), ces trois derniers éléments se verront néanmoins réduits à l'action, car la vie et le bonheur de l'homme se trouvent après tout, en cohérence avec la vision de l'homme développée dans l'éthique d'Aristote (EN I et II), dans son activité (Poétique 6, 1450a23). La tragédie est définie, in fine, comme mimesis d'actions.

Comme chez Platon, la mimétique permet l'apprentissage. Dans un tournant appréciatif, sans dire un mot sur ses dangers potentiels, Aristote

29. Si le texte n'est pas cohérent, mentionnant d'une part la troisième catégorie de gens qui sont semblables à nous (bomoious, 1448a6) et qui sont imités par quelques peintres, mais négligeant d'autre part totalement une troisième catégorie de drame, il faut rappeler qu'il s'agit d'un texte ésotérique (issu probablement d'un assemblage de notes de cours) qui n'était pas retravaillé pour être publié. 
la présente comme étant dans la nature humaine et comme source de la connaissance:

Dès l'enfance, le jeu mimétique est naturel à l'homme [to te gar mimeisthai sumphuton tois anthrôpois ek paidôn esti] (et l'homme diffère des autres animaux en ceci qu'il est plus mimétique qu'eux [mimètikôtaton], qu'il produit [poieitai] ses premières connaissances par le biais de jeux mimétiques [dia mimèseôs] $)^{30}$, et tous les hommes trouvent du plaisir aux imitations [kai to chairein tois mimèmasi pantas]. [...] En effet, nous aimons regarder les images [tas eikonas] parce que leur contemplation est suivie de l'apprendre [hoti sumbainei theôrountas manthanein] et nous concluons sur quelque chose de singulier [kai sullogizesthai ti hekaston] comme quand il y a le "c'est ça!" [hoion hoti houtos ekeinos] ${ }^{31}$.

Notons que non seulement la mimesis permet la connaissance, mais elle la produit ou la crée (poieitai). La mimesis, même celle de l'enfant qui joue, est donc un acte créatif dans le sens large de la poièsis.

En analysant le mimeisthai utilisé par Aristote dans la Poétique, Guénoun découvre un trait essentiel de ce terme qu'il essaie d'exprimer par «(re)présentation", suivant la traduction qu'en font Dupont-Roc et Lallot. Selon ces derniers:

mimeisthai + accusatif peut recouvrir deux relations bien différentes selon la nature de l'objet: le complément peut désigner l'objet-modèle [...] mais il désigne plus souvent, dans la Poétique, l'objet-copie, l'artéfact que l'on crée (Dupont-Roc/Lallot 156; Guénoun 20).

Ils traduisent le terme par «représenter» pour sa polyvalence comparable et Guénoun leur emboite le pas malgré le fait que ce terme caractérise plutôt, dans son livre, un autre régime. Or, le terme français «représenter " a deux défauts à mon sens. D'une part il est trop polyvalent, exprimant non seulement le jeu mimétique, mais aussi tout ce qui est d'ordre purement mental ou imaginaire. En l'absence de cet aspect, le terme «reproduire» serait à cet égard plus judicieux. D’autre part, il semble

30. Aristote, Poétique, 1148b4-9. L'homme se distingue d'autres animaux en ceci que d'une part les jeux d'enfants sont souvent des jeux d'imitation, davantage que chez les animaux; d'autre part, l'homme continue à jouer mimétiquement même à l'âge adulte.

3I. Aristote, Poétique 4, 1448b6-23; trad. Gernez modifiée; la modification s'efforce de rester proche des termes grecs. 
que «la copie», que le résultat donc soit plutôt présenté que représenté, davantage produit que reproduit.

Par contre, les termes «jouer» ou "mimer» combinent bien, en français, ces deux aspects: que ce soit un auteur qui écrit un dialogue avec Socrate, un metteur en scène qui met en scène un tel dialogue, ou un acteur qui joue Socrate, tous nous donnent «leur Socrate», leur «objet-copie» en faisant référence à Socrate comme "objet-modèle». Tout le monde, l'auteur, le metteur en scène et l'acteur aussi bien que le public, est conscient de ce double rapport - ou devrait l'être. Mais peut-être est-ce plus clair dans une comédie que dans une tragédie ou un dialogue socratique - le Socrate suspendu entre le ciel et la terre dans les Nuées est plus clairement différent du Socrate-modèle que le sont les Socrates des dialogues de Platon. On peut estimer plus haut le Socrate idéalisé de Platon que celui, plus réaliste, de Xénophon, comme le fait Sören Kierkegaard ${ }^{32}$. Bien sûr il y a d'une part l'intérêt historique, la volonté de savoir comment il était vraiment, ce Socrate. D’autre part, le jeu mimétique qu'a engagé Platon est, pour le dire avec Aristote, plus philosophique et plus sérieux que la simple volonté d'être fidèle aux faits, car il vise à exprimer une idée. La philosophie dépasse ici l'historiographie, elle dépasse la science en devenant poétique. La correspondance avec les faits et l'appropriation originelle constituent deux critères de qualité différents. N'est-ce pas justement dans la tension entre les deux qu'est situé le "vrai» d'une expression ou d'un jeu, et le "judicieux» d'une caricature?

On peut se demander, par conséquent, si le choix des termes "modèle» et "copie» que font Dupont-Roc et Lallot est bien approprié, car ce choix parait s'inscrire dans un régime qui présuppose, dans le jeu, en amont, la tentative d'arriver à la simulation exacte d'une chose ou d'une action, d'un caractère ou d'un personnage à reproduire. S'il s'agit plutôt d'un rapport d'expression il est, au contraire, plus adéquat de parler de «l'original» qui est "interprété» et du « résultat» que "donne» cette action mimétique.

Il est naturel pour l'humain depuis son enfance, dit Aristote, de jouer à des jeux imitatifs. Par ces jeux, qui reproduisent, certes, les comportements et les expressions de son entourage, il crée (poieitai)

32. Cf. S. Kierkegaard, Le concept d'ironie constamment rapporté à Socrate, première partie, ch. I. 
ses premières connaissances. Le théâtre comme art, peut-il être conçu comme prolongation collective de cette manière de créer des connaissances par les moyens des drames et de la scène?

\section{Denis Guénoun: le double besoin de la mimesis}

Frappé par l'observation des traducteurs, Guénoun conclut que «l'ambiguïté" de la mimesis par rapport à son objet grammatical et ontologique est structurale chez Aristote. Ce raisonnement semble juste, mais pourquoi conclure ensuite à "l'indifférence» du concept «à l'opposition entre la figure et son référent "? Cette ambivalence inhérente à la mimesis, n'était-elle pas évidente, présupposant ainsi la conscience du double jeu entre modèle et résultat? Un peuple dont un des dirigeants les plus adorés a dénoncé les contes d'Homère comme réfutés ${ }^{33}$, n'aurait-il pas une certaine conscience des déplacements (s'ils sont mensongers ou portés par d'autres mobiles) qui ont systématiquement lieu dans chaque expression, interprétation ou représentation? Ne s'agit-il pas du peuple qui a, avec l'invention de la comédie, appris à en rire? Loin d'être indifférente, la mimesis serait donc à définir par cette différence intrigante entre objet-original et objet-résultat; par la conscience de cette différence qui peut être source de curiosité, de joie, de méfiance, d'appréhension et finalement d'ironie et d'humour, ironie et humour dont Socrate et Aristophane sont sans doute les incarnations phares.

Le premier chapitre du livre de Guénoun se conclue sur le constat que «le système qu'il [Aristote] expose est aujourd'hui, irrévocablement, rompu " (41). C'est ici qu'un doute est permis. Car, même si le théâtre des Anciens est irrévocablement perdu, il y a un potentiel dans ces analyses inspirées par la Poétique d'Aristote que l'auteur de Pour le théâtre, merci Aristote reconnait très bien ${ }^{34}$.

33. Périclès dit d'Homère que ses "fabrications sont réfutées par la vérité" (Thucydide, Guerre, II.41).

34. Il vaut peut-être la peine de contextualiser cette prise de position de Guénoun, car elle pourrait concrétiser le passage fondamental dans la pensée récente de l'époque (post-)moderne à l'époque post-antique. Le Dreischritt historico-conceptuel opéré par Guénoun en 1997 est un mouvement typique de l'idéalisme allemand et de sa conception dialectique et évolutionniste de l'Histoire. Pour une telle pensée, il est normal de considérer l'approche des Anciens comme première étape à dépasser et déjà dépassée. 
"La vérité du texte théâtral est désormais, intempestivement, poétique» (p. 148) dit Guénoun du théâtre contemporain. Or, si nous devons comprendre la mimesis ancienne comme comportant un élément de création, comme j'ai essayé de le montrer, nous assistons aujourd'hui, dans le "régime du jeu", au retour de quelque chose de fondamental déjà articulé par les Anciens. Sous les couches d'interprétations hégémoniques, ne pourrait-on exhumer une approche restée couverte trop longtemps par une conception dualiste du théâtre comme (re)présentant ce qui a été, ou comme présentant ce qui devrait être, approche qui justifiait d'ailleurs excessivement le théâtre comme institution morale? Depuis un moment, nous ne pouvons plus simplement nous demander à quoi se réfère ce que nous voyons sur scène, car peut-être cela ne réfère-t-il à rien (cf. les pièces de Beckett). Pourtant, même dans ce cas, ce que nous percevons, cette absence de préexistant, de signifié, de représenté, est significative en elle-même. Elle nous rend conscient de ce qui se déroule autour de nous et, en cela, exprime davantage que ce que nous percevons dans la salle.

La singularité du théâtre serait donc d'exprimer des vérités sur l'homme et ses actions non sous forme de constats théoriques, comme le font les sciences humaines et sociales, mais de nous confronter avec une forme (ou structure interactionnelle) découverte par un auteur. Cette forme (du moins si elle est bien jouée) peut être comprise par les lecteurs, spectateurs et auditeurs (qui, d'ailleurs, se réjouiront du propos ou, au contraire, ne le supporteront pas). Le théâtre, ne serait-il pas ainsi la scène de la phronèsis, de la sagesse pratique, sagesse continuellement à refaire car à jamais imparfaite, ainsi que le dit Aristote? Le théâtre, serait-il le lieu d'une formulation, d'un partage et même d'une mise en jeu de notre compréhension du vivre ensemble? Un espace de découverte

Or, nous pouvons aussi considérer cette hypothèse évolutionniste comme dépassée et anachronique. Peut-être la préoccupation des Anciens a mené Guénoun, comme avant lui le Foucault tardif, à dépasser ses présupposés modernes (comme l'évolutionnisme), non pas pour passer à une étape post-moderne, mais à une pensée post-antique. Cette pensée est caractérisée par la conscience du fait que nous sommes imprégnés par les décisions conceptuelles des Anciens et par la volonté de questionner les anciens aiguillages pour se libérer d'éventuelles chaînes qu'ils nous ont imposées. 
collective de nouvelles structures d'une part, ou bien de l'actualité de constantes anthropologiques présumées d'autre part? ${ }^{35}$

Nous avons beaucoup parlé de valeurs cognitives et, si l'on suit les pas d'Aristote théoricien, il n'est pas étonnant que ce soit par ce biais qu'il organise sa défense dans le procès ouvert par Platon. Si par contre, quelqu'un soutenait qu'en réalité, le théâtre est davantage l'affaire de l'émotionnel que du cognitif, et demande où trouver l'émotionnel dans cette approche aristotélicienne, la réponse ne serait pas non plus à trouver dans la catharsis. Comme Guénoun le montre bien, c'est, dans le texte aristotélicien, la découverte elle-même qui procure du plaisir chez le public. Le plaisir est là: dans la "transmission" possible d'un «c'est ça!», dans l'expression (et non la copie) d'une généralité sur nos interactions libres par le biais d'actes et de paroles concrets, expression telle que la lectrice, la spectatrice et l'auditrice puissent juger: "Oui, c'est cela qui nous arrive! C'est cela que nous faisons!» Et ce jugement met en lien le général qu'elle perçoit dans le concret des actions scéniques avec les singularités de son propre vécu. On peut se tromper: l'auteure le peut, l'actrice le peut, la spectatrice le peut aussi. Mais parfois, la perception du crucial nous frappe. A cet égard, affect et découverte ne font qu'un. Le bouleversement ressenti dans le "c'est ça!» ne mène pas nécessairement à une explication rationnelle du saisi. L'homme est une entièreté; $s$ 'il est plus mimétique que les autres animaux, cela veut aussi dire que sa production ludique de connaissances ne se fait pas sans affects ni jouissance ${ }^{36}$.

Le mérite principal de l'analyse de la Poétique effectuée par Guénoun est clairement d'avoir mis en lumière l'importance de la connaissance, le "celui-là, c'est lui» (dans la traduction qu'il utilise). Il met en évidence qu'il ne s'agit pas, à cet endroit, d'une re-connaissance qui ne serait

35. Ce qui expliquerait pourquoi un Sophocle, un Shakespeare, un Molière ou un Schiller sont toujours joués. Voir à cet égard la contribution de David Bouvier. Mais un doute s'installe à cet endroit: au lieu de parler d'expression, ne vaudrait-il pas mieux parler de partage? Car en ce qui concerne le théâtre ou l'écriture, l'expression vise toujours le partage (Mitteilung) dans un sens communicationnel. L'expression d'une chose n'est pas sans une volonté de la dire ou de la montrer à quelqu'un. Si j'opte néanmoins pour le terme expression, ce n'est pas par volonté de négliger l'aspect de partage, mais pour créer un contraste clair entre la mimesis comme activité poétique au sens large (c'est-à-dire productive) et l'imitation dans le sens de simulation ou de copie.

36. Voir à cet égard la contribution de Vincent Laughery qui semble, par un itinéraire différent, en expliquant la différence entre l'émotion et l'affect, arriver à la même conclusion. 
qu'une identification - l'anagnorisis figure ailleurs, plus tard dans la Poétique -, mais d'une connaissance active, d'une découverte, comme lorsqu'on est frappé par un portrait qui exprime mieux que chaque instant réel la personnalité d'une personne: «ça, c'est elle!», nous faisant ressentir que "c'est juste», que "c'est vrai».

C'est le point crucial de l'analyse de Guénoun. Le spectateur (ou lecteur) découvre lui-même une forme et juge si oui ou non elle s'applique. Si Aristote parle de l'intrigue comme de l'âme du drame, c'est que le drame est davantage qu'une simple action, il est plusieurs actions, il contient plusieurs caractères et pensées, qui donnent ensemble quelque chose de général à voir - comment nommer ce quelque chose: forme? structure? Les drames nous montrent ce qui peut arriver si plusieurs volontés, actions, intentions et caractères entrent en conflit. Ils montrent les résultats généraux de nos interactions libres.

Rappelons encore les dernières remarques de Guénoun qui méritent à mon sens d'être mises en valeur et fourniraient un point de départ fertile pour des recherches ultérieures: il pose la question de la nécessité du théâtre. La réponse est un double "oui» ancré dans la Poétique d'Aristote: l'homme a "besoin de représenter, besoin de regarder ce qui se représente " ${ }^{37}$. Or, les deux besoins figurent sans lien essentiel chez Aristote et ce lien serait donc à développer dans une philosophie de la scène:

On peut penser que cette union procède, logiquement, de ce que cette pratique et cette théorie s'impliquent l'une l'autre. La pratique ne se suffit pas de son auto-effectuation, il lui faut se présenter à un regard qui dégage et découvre ses formes intelligibles ${ }^{38}$.

Voilà l'essentiel - sous condition que la "forme» ne traduise pas l'idée de Platon, mais la morphè ou "le général» d'Aristote, englobant les vérités anthropologiques aussi bien que les structures qui émergent et disparaissent dans les mouvements de l'Histoire ou plutôt: des Histoires, et des cultures. Voilà l'essentiel - et pourtant... :

La Poétique témoigne, à nos yeux rétifs, de ce théâtre de praticiens et théoriciens ligués. [...] Ce théâtre, en aucune façon, ne peut être

37. D. Guénoun, Le théâtre est-il nécessaire?, p. 40.

38. Ibid., p. 41. 
encore le nôtre. Et la nécessité, éventuelle, de notre théâtre ne peut aucunement résulter de ses dispositions ${ }^{39}$.

Si les importantes observations de Guénoun mériteraient d'être poursuivies dans les recherches à venir ${ }^{40}$, je suis sceptique par rapport à sa conclusion qui relativise les résultats de son analyse de la Poétique en les reléguant à une époque passée. Elle bloque l'application de son analyse à l'écriture dramatique et au jeu contemporains, mais pour quelle raison? Faut-il lire ici des présupposés (post-hégéliens) et notamment la supposition d'une évolution historique en correspondance avec une logique de l'esprit? Le jugement, sous-entend-il une attitude moderniste? Pourtant, le défi actuel n'est plus de dépasser les analyses dichotomiques du passé pré-Lumières (y compris, à l'occasion, celles du classicisme français), défi caractéristique de l'époque moderne. Ce défi n'est pas non plus, d'ailleurs, post-moderne, mais bien plutôt post-antique: il ne s'agit pas de s'inscrire dans un processus évolutif (présupposé) en marche depuis les Grecs, mais au contraire de se libérer des chaînes que les Anciens ont parfois imposées à la pensée (si bonnes qu'aient pu être leurs intentions). En ce sens, nous passons, depuis des décennies déjà, à l'époque postantique. Le fait que le théâtre de nos jours soit loin des tragédies et des comédies des anciens Grecs n'empêche pas de trouver des éléments dans la pensée aristotélicienne du drame qui nous permettent de nous libérer des entraves par lesquelles un certain platonisme a attaché la conception de l'art aux idées éternelles.

\section{Michael Groneberg \\ Université de Lausanne}

39. Ibid., p. 41 sq.

40. Elles donnent, en outre, une réponse au problème soulevé par Schaeffer selon lequel «les faits que je viens de présenter montrent seulement que la mimésis ludique peut avoir une fonction cognitive pour celui qui crée des imitations» (Pourquoi la fiction?, p. 131) mais pas pour les spectateurs ou lecteurs car ils ne produisent pas de mimèmes. L'analyse de Guénoun, pas encore mentionnée par Schaeffer, montre des voies pour expliquer que les spectateurs aussi, et non seulement les acteurs, profitent cognitivement d'un spectacle. 


\section{BIBLIOGRAPHIE}

\section{Textes}

Aristote, Catégories/De l'interprétation, éd. bilingue, trad. Catherine Dalimier, Paris, Flammarion, 2007.

—, Éthique à Nicomaque, trad. Richard Bodëus, Paris, Flammarion, 1997.

—, Poétique, trad. Roselyne Dupont-Roc, Jean Lallot, Paris, Seuil, 1980.

—, Poétique, éd. bilingue, trad. Barbara Gernez, Paris, Les Belles Lettres, 2008.

Gorgias, Die Fragmente der Vorsokratiker, 3 Bd., hrsg. v. Hermann Diels, Walther Kranz (10 éd.), Berlin, Weidmann, 1960-1961 (Les Présocratiques, trad. Jean-Paul Dumont, Paris, Gallimard, 1988).

Platon, Le Banquet, trad. Luc Brisson, Paris, Flammarion, 1999.

—, République, trad. Robert Baccou, Paris, Flammarion, 1966.

—, Symposium, in "Salut Socrate!» Le Symposium de Platon adapté pour la scène, nouvelle trad., éd. et commentaires par Michael Groneberg, Lausanne, CTL, 2010.

Plotin, Ennéades, tome V, trad. Emile Bréhier, Paris, Les Belles Lettres. 1931.

—, Traités, tome V, trad. Luc Brisson et Jean-François Pradeau, Paris, Flammarion, 2006.

Thucydide, La Guerre du Péloponnèse, trad. Jacqueline de Romilly, Paris, Laffont, 1990.

\section{Travaux}

Brisson, Luc, "Logos et logoi chez Plotin. Leur nature et leur rôle", Les Cahiers philosophiques de Strasbourg, 8 (1999), p. 87-108. 
Dupont, Florence, Aristote ou le vampire du théâtre occidental, Paris, Aubier, 2007.

Genette, Gérard, "Frontières du récit», in Figures II, Paris, Seuil, 1969. GuÉNoun, Denis, Le théâtre, est-il nécessaire?, Circé, 1997.

Jouvet, Louis, Témoignages sur le théâtre, Paris, Flammarion 2002 [1951].

KierkegaArd, Sören, Le concept d'ironie constamment rapporté à Socrate, Paris, Editions de l'Orante, 1975 [1841].

Puelma, Mario, Der Dichter und die Wahrheit in der griechischen Poetik von Homer bis Aristoteles, Universitätsverlag Freiburg Schweiz, 1989.

Schaeffer, Jean-Marie, Pourquoi la fiction?, Paris, Seuil, 1999.

Schniewind, Alexandrine, L'éthique du sage chez Plotin. Le paradigme du spoudaios, Paris, Vrin, 2003.

Schopenhauer, Arthur, Le monde comme volonté et comme représentation, Paris, PUF, 1966 [1819, 1859³]. 
